\title{
Aplicação da ferramenta stage-gate no gerenciamento de projetos de máquinas para Indústrias moveleiras
}

\author{
Vanderlei Cieslinski \\ vcieslinski1984@gmail.com \\ Universidade da Região de Joinville \\ (UNIVILLE), São Bento do Sul, Santa \\ Catarina, Brasil \\ Diego Alves de Miranda \\ diegoalves_klx@hotmail.com \\ Universidade da Região de Joinville \\ (UNIVILLE), São Bento do Sul, Santa \\ Catarina, Brasil
}

\begin{abstract}
RESUMO
As indústrias fabricantes de máquinas seja de pequeno, médio ou grande porte, precisam estar preparadas para dar respostas imediatas com projetos altamente tecnológicos e em prazos curtos para as necessidades identificadas no mercado. Existem ferramentas de gerenciamento de projetos que podem trazer inúmeros benefícios para as empresas nestes quesitos. $\mathrm{E}$ o tempo de resposta pode determinar a permanência de uma empresa no mercado ou não. Este trabalho demonstra a aplicação de uma metodologia de gerenciamento de projetos tendo por base a ferramenta Stage-Gate com documentos relacionados a áreas do $P M B O K$ no desenvolvimento de uma máquina específica para o mercado moveleiro, identificada como necessidade para o mercado mundial. O trabalho teve por base um estudo de caso, no qual demonstrou a importância da implementação de uma metodologia de gerenciamento de projetos para o desenvolvimento de máquinas para o setor moveleiro. A análise da aplicação deste tipo de metodologia permitiu ter uma visão mais abrangente do projeto, podendo identificar possíveis dificuldades com antecedência, permitindo a ação e correção dos eventuais problemas, que venham a prejudicar o resultado final do projeto.
\end{abstract}

PALAVRAS-CHAVE: Gerenciamento de Projetos. Stage-Gate. PMBOK. Máquinas para Indústria Moveleira. 


\section{INTRODUÇÃO}

Com os desafios da globalização, as organizações vêm constantemente sentindo a necessidade da aplicação de ferramentas do planejamento e gestão de suas atividades, isso é necessário para se manter no mercado de forma competitiva. Entre as inúmeras ferramentas destaca-se a de planejamento estratégico, que auxilia as empresas nas tomadas de decisão bem como ajuda a alcançar os seus objetivos Alves et al., (2013). Coutinho e Ferraz (1994), afirmam que a competitividade tratada de um ponto de vista dinâmico, pode ser a oportunidade de uma empresa individualmente ou com parceiros comerciais, desenvolver estratégias concorrenciais que permitam a sua permanência de forma duradoura e sustentável no mercado.

Para Mello et al., (2012), com este mercado altamente competitivo se torna fundamental a busca por inovação e uma boa condução dos processos, tanto de gerenciamento estratégico como os de desenvolvimento de novos produtos pode permitir a excelência no desempenho das organizações, importante salientar que o processo de inovação competitiva é muito mais que simplesmente ter boas ideias. Rozendeld et al., (2006) explicam que dentro das organizações é indispensável conter processos bem estruturados, para criação de novos produtos mais competitivos, atendendo prazos e custos pré-estipulados pelo mercado, permitindo assim a permanência ou mesmo ampliação de sua participação no mercado.

Segundo Seveto et al., (2014), a inovação pode ser considerada como um dos elementos vitais para as organizações pois tanto os indicadores de gastos com pesquisa e desenvolvimento como o de desempenho das empresas estão correlacionados, ressaltando assim a importância do processo de inovação para a criação de vantagens competitivas. Para se ter uma vantagem competitiva as organizações devem agregar a suas habilidades, a aplicação de ferramentas que auxiliem na exploração das oportunidades identificadas no mercado, oportunidades que pode ser por exemplo o desenvolvimento e introdução de um novo produto (BESANKO et al., 2000).

Entre as várias ferramentas que podem auxiliar as organizações tem-se a Stage-Gate, que é uma importante ferramenta de gerenciamento de projetos, podendo ser constituída por várias ferramentas auxiliares para o desenvolvimento de novos produtos. Para Mello et al., (2012), a utilização da ferramenta Stage-Gate pode trazer muitos benefícios para organização, como por exemplo, resultados mais rápidos sobre o investimento, obtido com menores ciclos de desenvolvimento e uma maior integridade multifuncional, isso se torna possível se a ferramenta for aplicada no momento da identificação de um novo produto ou serviço no mercado. Esta ferramenta vem auxiliando em diversas áreas da economia mundial, pois de acordo com Burgwal et al., (2018) esta fermenta vem sendo inclusive aplicada na melhoria do processo de inovação e desenvolvimento de novas vacinas, que devido a baixos investimentos em pesquisa e também a falta de um conjunto de etapas que gerenciem o processo, vem apresentando uma série de deficiências para o atendimento das demandas de mercado.

Paluch (2019), trata o Stage-Gate dentro do desenvolvimento ágil na era digital ressaltando que o princípio básico do Stage-Gate é a redução dos riscos que envolve o processo de inovação, onde existe um número de portas para 
tomada de decisões que determinam se o projeto estudado segue a diante ou para naquele momento, essas decisões são tomadas com base em indicadores de desempenho predefinidos.

Nesta contextualização se percebe a importância de uma ferramenta para o desenvolvimento de projetos, sendo assim, o objetivo do presente trabalho é analisar e mapear a utilização da ferramenta Stage-Gate no desenvolvimento específico de uma máquina para a indústria moveleira. Setor da economia que vem vivenciando na íntegra as mudanças de mercado e necessita de máquinas para uma maior produtividade com qualidade, para continuar sendo competitivo diante de uma economia agressiva e de alcance global. Para isto, foi preciso entender o que é a necessidade de mercado, foram mapeadas as etapas do Stage-Gate e documentadas, para desenhar um ciclo de desenvolvimento de um projeto de máquina.

\section{DESENVOLVIMENTO DE PROJETOS}

As empresas que atuam em suas atividades normais com base em áreas de desenvolvimento ou propriamente dito projetos de produtos, vivenciam um ambiente extremamente complexo e dinâmico comparado com as empresas tradicionais que priorizam apenas a fabricação de bens ou bens de serviços. Consequentemente a administração dessas empresas exigi um bom planejamento estratégico com uma abordagem diferenciada para a Administração de Projetos, a qual utiliza de formas organizacionais e específica técnicas avançadas de controle (FILHO et al. 1999).

Atualmente existe uma serie de variáveis ligadas ao gerenciamento de um projeto podendo ser elas ferramentas de gerenciamento ou mesmo a própria caracterização de uma necessidade identificada, neste capitulo abordaremos ferramentas para o gerenciamento de projetos como Stage-Gate e PMBOK e as particularidades da necessidade de mercado quando identificada.

\section{STAGE-GATE}

Uma das ferramentas de planejamento estratégico na administração de projetos da empresa é a Stage-Gate, que de modo geral permite a introdução de suas técnicas em diversas áreas de uma mesma empresa. A necessidade de ferramentas como está no gerenciamento de projetos já é identificada a muito tempo, sendo já mencionada por Rabechini Jr et al., (2002), descrevendo que no Brasil, existia um número reduzido de empresas que têm um modelo de gerenciamento de projetos desenvolvido, enaltecendo ainda que a ferramenta Stage-Gate poderia dar suporte para implantação de novas metodologias de gerenciamento de projetos na época. No entanto podemos perceber que no decorrer do tempo a procura e utilização de tal ferramenta se fez mais presente pois conforme Santos et al., (2012), o número de empresas que abordam técnicas de gerenciamento de projetos obteve um crescimento expressivo nos últimos anos, se percebendo assim o aumento das tendências de utilização do gerenciamento de projetos como ferramenta administrativa e estratégica.

De acordo com Cooper (2008), a ferramenta Stage-Gate (Figura 1), pode ser considerada como um mapa que tem por objetivo guiar os projetos de 
desenvolvimento de novos produtos, isso desde a ideia inicial chegando até o lançamento do produto ao mercado. Desta forma pode ser caracterizado como um modelo para auxiliar a gestão do processo de desenvolvimento de novos produtos.

Figura 1 - Esquema do modelo Stage-Gate

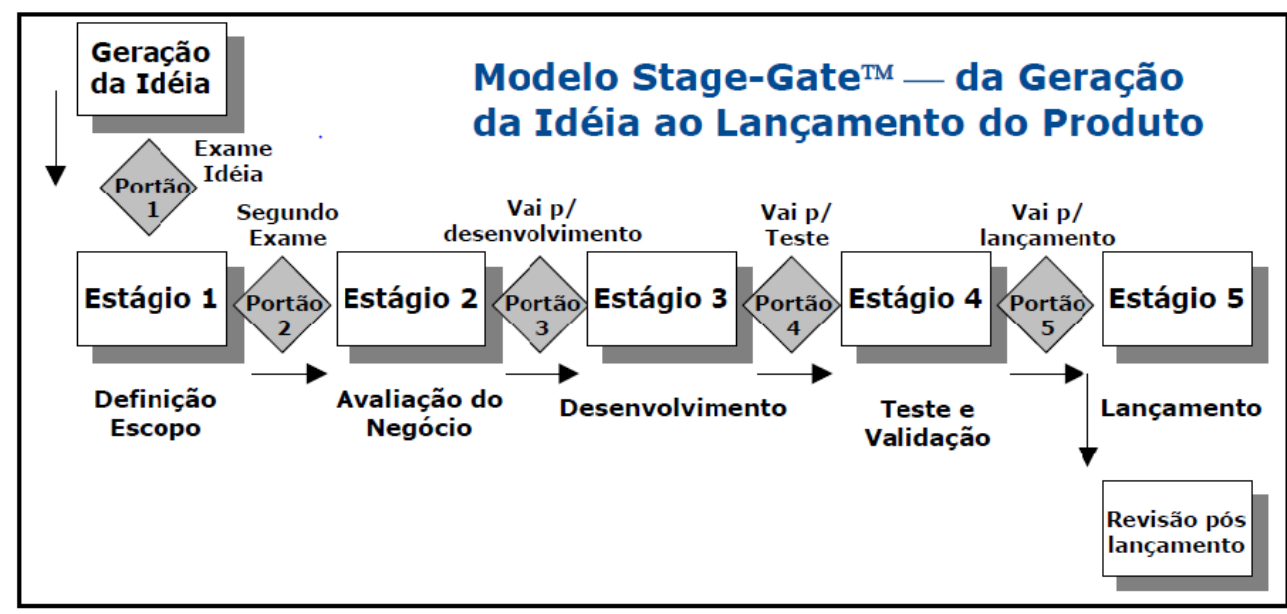

Fonte: Cooper (2008)

Segundo Cobaito (2013), a maior contribuição e o grande desafio da ferramenta Stage-Gate dentro do gerenciamento de projeto é a identificação dos Gates, que servem como pontos de decisão para dar sequência ou bloquear um projeto. Sendo os Gates um dos fatores de maior impacto no sucesso do projeto, uma boa definição desses Gates pode levar a uma tomada de decisão mais precisa e permitindo a continuação do projeto de desenvolvimento de produto.

Os processos de desenvolvimento de novos produtos utilizando o método Stage-Gate fornecem inúmeras vantagens competitivas para as empresas, pois a concorrência não terá capacidade de alcançar êxito no desenvolvimento de um produto com a mesma rapidez e com o mesmo nível de qualidade se não utilizar das mesmas técnicas. Na utilização desta ferramenta projetos que representem baixo potencial de retorno são descartados rapidamente pela empresa no início de seu desenvolvimento (SEVERO et al., 2014).

\section{PMBOK}

Também como auxílio para o gerenciamento de projetos foi criado no ano de 1969 o Project Management Institute (PMI), que atua no melhoramento do processo de gestão de projetos direcionado diretamente ao surgimento de novos negócios e mercados, que são normalmente orientados por encomendadas, rapidez nas inovações tecnológicas e competitividade crescente dentro das organizações seja ela internamente ou externa. Dentro deste instituto surgiu a importante ferramenta de gerenciamento de projetos denominada Project Manangement Body of Knowledge - PMBOK (PMI, 2019).

Para Santos et al., (2012), gerenciamento de projetos abrange uma aplicação de conhecimentos, habilidades e ferramentas específicas que possibilitam entender os requisitos de uma necessidade que posteriormente será utilizado no desenvolvimento de um projeto. Conforme PMI (2019), a utilização desta 
contextualização de gerenciamento de projetos estipulados pela $P M B O K$ pode ser aplicada em uma grande variedade de projetos que podem ser definidos como um conjunto de atividades temporárias, realizadas em grupo e destinadas a produzir um produto, serviço ou resultados únicos.

$O$ guia de gerenciamento de projetos $P M B O K$ é dedicado as melhores práticas para o Gerenciamento de Projetos. Sua macroestrutura são 47 processos que abrange dez áreas de conhecimento sendo agrupados em cinco grupos de processos, esses processos recebem as entradas e com auxílio de ferramentas produzem documentos que permitem uma saída para uma próxima etapa, como trata-se de um processo de gestão ele deve ser flexível e normalmente precisa de adaptações de acordo com as características individuais de cada projeto (MELO et al., 2017). Na Figura 2 estão destacadas as áreas de conhecimento tratadas pelo $P M B O K$ e os processos distribuídos de acordo a divisão dos grupos.

Figura 2 - Área de conhecimentos e grupos de processos do PMBOK

\begin{tabular}{|c|c|c|c|c|c|}
\hline \multirow{2}{*}{$\begin{array}{c}\text { Áreas de } \\
\text { Conhecimentos }\end{array}$} & \multicolumn{5}{|c|}{ Grupos de Processos } \\
\hline & Iniciação & Planejamento & Execução & Controle & Encerramento \\
\hline Integração & ${ }^{*}$ & ${ }^{*}$ & ${ }^{*}$ & kt & ${ }^{*}$ \\
\hline Escopo & & \#たネ & & ** & \\
\hline Tempo & & $\begin{array}{l}\text { たたたたた } \\
\end{array}$ & & ${ }^{*}$ & \\
\hline Custo & & \#末 & & ${ }^{*}$ & \\
\hline \begin{tabular}{|l|} 
Qualidade \\
\end{tabular} & & ${ }^{*}$ & ${ }^{*}$ & ${ }^{*}$ & \\
\hline Recursos humanos & & ${ }^{*}$ & \#末 & & \\
\hline Comunicaçăo & & ${ }^{\star}$ & ${ }^{*}$ & ${ }^{*}$ & \\
\hline \begin{tabular}{|l|} 
Risco \\
\end{tabular} & & 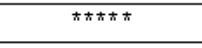 & & ${ }^{*}$ & \\
\hline Aquisiçăo & & ${ }^{*}$ & ${ }^{*}$ & ${ }^{*}$ & ${ }^{*}$ \\
\hline Partes interessadas & ${ }^{*}$ & ${ }^{*}$ & ${ }^{*}$ & ${ }^{*}$ & \\
\hline Total de Entregas & 2 & 24 & 8 & 11 & 2 \\
\hline
\end{tabular}

*Entregas de cada área de conhecimento por Grupo de Processos

Fonte: Adaptado de Melo (2017)

Como pode-se verificar na Figura 2, existe uma correlação importante entre as áreas de conhecimento e os grupos de processos, destacando que no planejamento ocorre a maior quantidade de entregas ou processos, sendo assim reforçado a abordagem de Rodrigues (2016) que defende que a estratégia e o planejamento gerencial seja da organização ou de um possível desenvolvimento é indispensável para reforçar a posição da organização no mercado, promovendo a satisfação dos clientes e atingindo os objetivos.

\section{NECESSIDADE DE MERCADO}

Atualmente ocorre um processo altamente evolutivo que gera necessidades de mercado constantes nos mais diversos segmentos da economia, por este motivo as organizações devem esta atualizadas para evitar perca de tempo seja no planejamento ou na implantação de um novo produto ou serviço no mercado. Além da evolução natural proporcionada pelas altas tecnologias desenvolvidas existe as questões competitivas em um cenário de muitos concorrentes preparados para atender as mesmas necessidades identificadas.

Tendo como acirrada as concorrências atuais entre as organizações, o termo estratégia anteriormente utilizado apenas para práticas militares, foi instituído 
nos setores administrativos das indústrias, com o propósito de conseguir sucesso nas batalhas gerais (ALVARES et al., 2018).

Se avaliarmos os desafios que o mercado exige das empresas e de caráter normal que seja adorado uma postura estratégica com relação aos negócios, a compreensão da importância dos clientes é requisito fundamental para que a organização tenha um crescimento tendendo a melhorar seus números de venda, conquistando assim novos consumidores e oferecendo produtos, serviços e atendimentos de qualidade (COSTA, 2015).

\section{METODOLOGIA}

Como este trabalho produz a análise da aplicação da ferramenta Stage-Gate no desenvolvimento de uma máquina para indústria moveleira, a metodologia foi dividida em etapas para que se alcance os objetivos com êxito. As atividades foram baseadas na literatura e a coleta de dados e agrupamento de documentos utilizados durante o processo de desenvolvimento foram de suma importância para a compilação das informações.

\section{ENTENDIMENTO DA ATUAL NECESSIDADE NO MERCADO}

Diante do atual mercado moveleiro as empresas buscam constantemente novos equipamentos para seus parques fabris, permitindo assim aplicar mais tecnologia e agregar mais valor aos seus produtos. Para Sperotto (2018), a grande parte das empresas deste setor interpretam a inovação tecnológica que está inserida nos materiais utilizados, assim como nos bens de capital como máquinas, equipamentos e ferramentas a grande relevância para se manter no mercado e tratando-se de máquinas, existe uma variedade enorme destinado para as mais diversas funções.

O levantamento da necessidade ou do equipamento que foi aplicado a ferramenta Stage-Gate foi realizado por uma equipe comercial responsável pelas vendas de máquinas, e também através de pesquisa realizada por profissionais denominados gerentes de produto responsáveis pela identificação, documentação e apresentação de uma possível necessidade. No presente artigo será levantado quais são as principais etapas do desenvolvimento de produtos.

\section{MÉTODO PRIMITIVO DE GERENCIAMENTO DE PROJETOS DE MÁQUINAS}

O desenvolvimento de um novo produto em qualquer organização segue um fluxo que obrigatoriamente deve respeitar etapas a serem realizadas de acordo com cada área da empresa e suas responsabilidades. Sem a aplicação de uma ferramenta de gestão no desenvolvimento de um projeto podemos ter apenas uma lógica pré-determinada sem um controle dos processos para execução de novos projetos, sendo esta a situação inicial para o desenvolvimento de produtos na empresa estudada.

De acordo com Salgado (2010), para se ter um controle eficaz da pesquisa e desenvolvimento de novos produtos é necessário realizar a descrição das atividades dos estágios e do processo de desenvolvimento de produto como um 
todo, isso exige que seja feito uma estruturação de forma ordenada das etapas para a visualização das características especificas no desenvolvimento de produto de cada empresa. Na Figura 3 é representada as etapas do desenvolvimento de um novo produto na empresa, a qual foi aplicada este estudo, detalhando a divisão das atividades é de acordo com os setores e suas competências.

Figura 3 - Mapeamento das etapas do Stage-Gate e da documentação

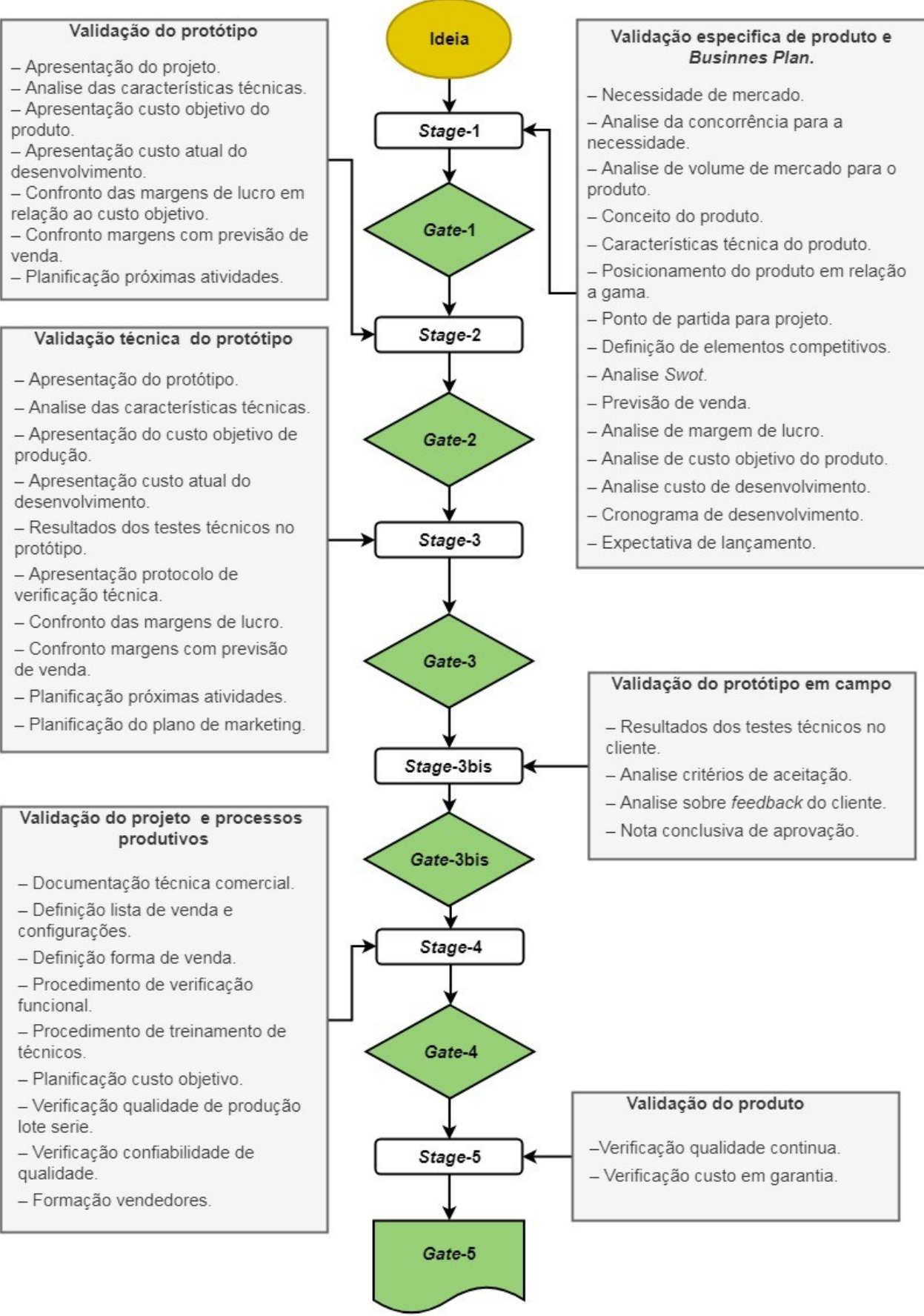

Fonte: Autoria própria (2019)

O fluxo de desenvolvimento da empresa se sustenta em um processo parcialmente contínuo, pois em algumas etapas ele pode parar e retornar para etapas anteriores, porém este fluxo ainda não avia sido desenhado de forma 
clara e visível para todos os envolvidos nos processos, então para um melhor entendimento das fases do desenvolvimento de um produto na empresa foi realizado um ciclo em forma de fluxograma das etapas do desenvolvimento de produto, listando a etapa suas entradas e saídas conforme demonstra a Figura 4.

Figura 4 - Fluxograma de desenvolvimento de produto (antes do Stage-Gate)

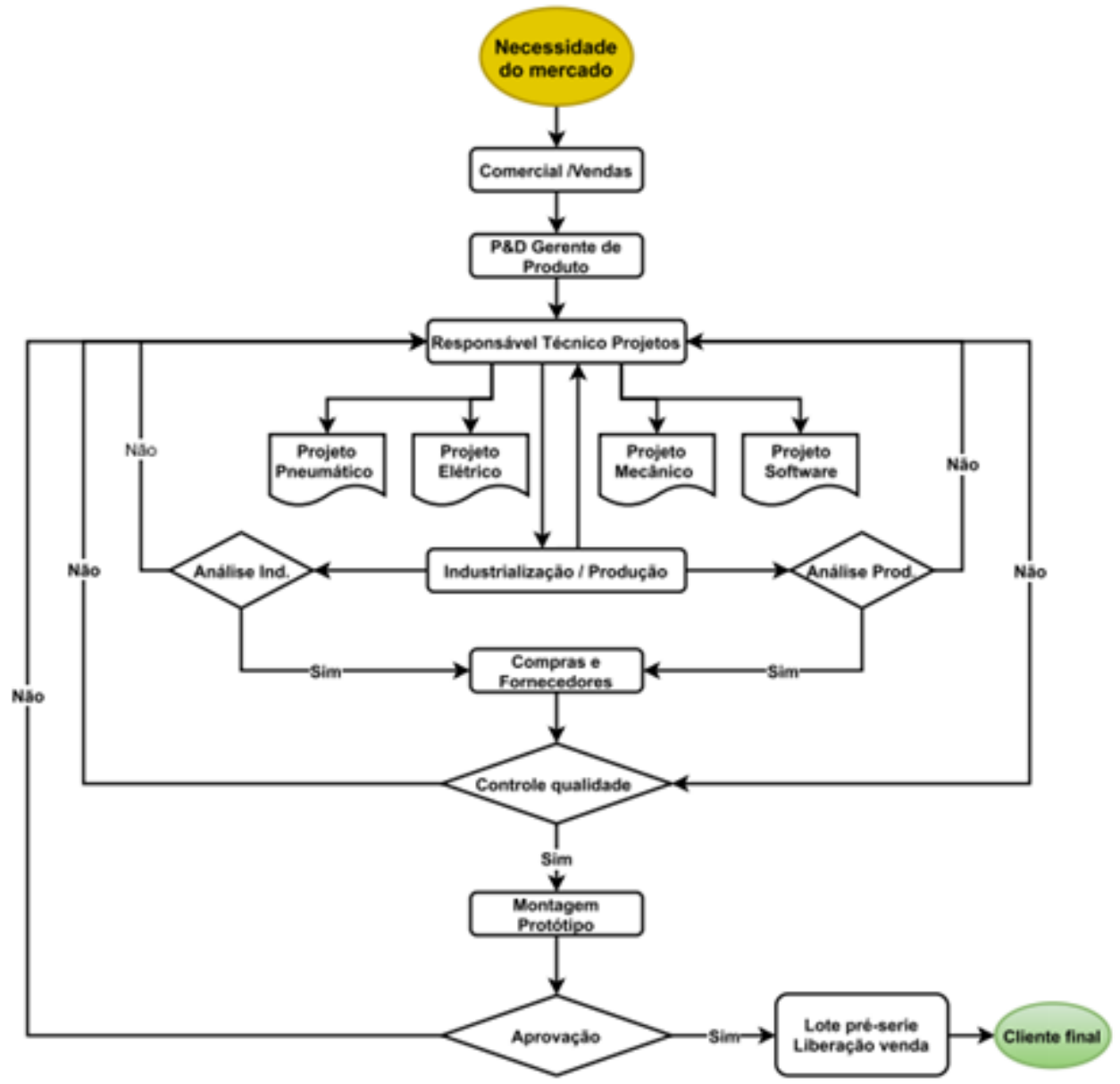

Fonte: Autoria própria (2019)

A necessidade de mercado é o ponta pé inicial para DNP (desenvolvimento de novos produtos) e surge por uma tendência de mercado que é identificada pelo cliente representantes ou mesmo por um gerente de produto os quais estão mais presentes no mercado e tem uma percepção maior das possíveis necessidades que poderão surgir.

As informações colhidas no mercado são passadas diretamente para o setor de P\&D (pesquisa e desenvolvimento) onde atua os profissionais denominados gerentes de produto, que são dentro de uma organização os verdadeiros mentores da implantação de novos produtos no mercado, assim como a linha direta de comunicação entre necessidade de mercado e setor de desenvolvimento do produto que é representado no fluxograma como responsável técnico do projeto.

A parte de desenvolvimento de produto é dividida em quadro macro áreas que é o projeto mecânico, projeto elétrico, projeto pneumático e projeto de software essas áreas em geral recebe informações diretamente do responsável 
técnico do projeto que no cenário atual trata-se de um dos integrantes responsável pelo desenvolvimento do projeto mecânico das máquinas.

Com a finalização da parte de desenvolvimento do produto, iniciam-se os trabalhos da industrialização do produto que tem como principal objetivo o planejamento de produção, compra ou beneficiamento de todos os componentes e recursos necessários para a fabricação e montagem do equipamento. A industrialização é uma área muito ligada ao setor de produção da empresa e tem grande influência na aprovação de um projeto, os trabalhos em conjunto entre engenharia do produto, industrialização e produção são responsáveis por fabricar uma determinada máquina no custo e na qualidade estabelecida na identificação da necessidade.

Os processos da sequência consistem na parte de programação e compras das peças e componentes sendo eles comerciais ou fabricados, e na sequência é produzido e montado o protótipo, onde é estabelecido que para um bom andamento da montagem e do funcionamento do protótipo todo item produzido pela primeira vez na organização e que vai dar origem a uma nova máquina deve obrigatoriamente passar pelo setor de qualidade responsável por conferir se as peças estão todas dentro das características técnicas e dimensionais desejadas.

A montagem do protótipo e aprovação é considerada como a fase mais crítica no fluxo de desenvolvimento de um novo projeto é onde aparecem as reais dificuldades e oportunidades de melhoria na nova máquina desenvolvida. Nesta etapa é verificado se o equipamento irá atender todos os requisitos indicados na descoberta da necessidade, se o projeto está dentro das características necessárias para produção, se a máquina está aprovada tecnicamente na fase de protótipo atendendo a custos prazos e qualidade, estes são os gatilhos principais e que determina se a máquina irá entrar em produção, permitem a liberação da fabricação do lote pré-série.

A liberação da máquina para a produção de um lote pré-série é o momento que a produção se adequa para produzir a nova máquina e se inicia toda as etapas de divulgação preparação de equipe de vendas, pois após a fabricação e montagem do lote pré-série será autorizado a venda deste equipamento permitindo assim atender à necessidade indicada no mercado.

\section{IDEALIZAÇÃO DO APRIMORAMENTO NO DESENVOLVIMENTO DE MÁQUINAS}

Foi realizada uma reunião com gerentes de produto e gerente de Engenharia que são responsáveis pelo fluxo de desenvolvimento de novos produtos da empresa, tendo como objetivo a planificação das etapas do Stage-Gate aplicado ao desenvolvimento da máquina especifica. Nesta reunião foram listadas as principais etapas bem como suas entradas e saídas, iniciando com a necessidade identificada do projeto e chegando ao lançamento do produto no mercado que finaliza o primeiro ciclo de desenvolvimento. Conforme Fosi (2016), a utilização do Stage-Gate pode ser um importante fator dentro das organizações e a concretização das etapas bem como sua documentação são fundamentais para atingir o sucesso do projeto. 
Na Figura 3 é demonstrado no formato de fluxograma as etapas do StageGate onde é listado as entradas ou documentos necessários em cada etapa a compilação de desses documentos é a chamada Stage, processo que antecede a realização do Gate de aprovação do projeto.

Segundo Gomes e Souza (2010), o mapeamento de processos dentro de uma organização é uma importante ferramenta gerencial e de comunicação que pode trazer muitos benefícios e têm a intenção de auxiliar na melhoria dos processos existentes ou mesmo na implantação de uma nova estrutura baseada em fluxo de processos. As vantagens da aplicação permitem a redução de custos na pesquisa e desenvolvimento de novos produtos e serviços e também a redução de falhas permitindo a integração entre sistemas e melhorias no desempenho da organização.

\section{APLICAÇ̃̃O DE STAGE-GATE}

Baseado nas etapas de DNP atribuídos na Figura 4 foi possível inserir as etapas da ferramenta Stage-Gate (Figura 3), priorizando uma divisão dos cinco macros atividades denominadas Gates da ferramenta de forma uniforme tendo abrangência em todos os processos de desenvolvimento de produto atualmente utilizado pela empresa.

A introdução dos Gates dentro do fluxo de desenvolvimento de produto utilizado permitiu uma melhor divisão das atividades e também evidenciou onde são os pontos chaves para tomada de decisão durante um desenvolvimento de um projeto de máquina. Como é uma característica dentro de qualquer processo de gestão a tomada de decisão com base em dados ou números, foi separado os documentos necessários para a tomada de decisão em cada Gate e direcionado indicado qual a área é responsável pela compilação de tal documento.

Com base nos estudos propostos e buscando os melhores resultados com a aplicação da ferramenta Stage-Gate, utilizou-se uma necessidade de uma máquina diferenciada para corte de chapas de madeira, necessidade esta que foi identificada no mercado moveleiro.

A necessidade de mercado de uma máquina para corte de chapas de madeira contendo as funções de corte reto 90 e e corte 45 - em relação a base da máquina. Esta necessidade surgiu na Europa, devido ao pouco espaço nas empresas não permitindo que as mesmas adquirissem as atuais seccionadoras horizontais para corte 90 e e as serras circulares para corte 45․ Nesta nova proposta, o mesmo equipamento irá fazer as duas furações. Entretanto, o desenvolvimento do projeto desta nova máquina será testado a aplicação do Stage-Gate.

\section{RESULTADOS E DISCUSSÕES}

A utilização de uma nova ferramenta em um processo novo pode ser considerada simples pelo fato do processo se adaptar à nova ferramenta. Porém, quando o objetivo é a aplicação de uma ferramenta nova em um processo já existente em muitos casos exige pequenas adaptações até mesmo na ferramenta a ser implantada, e uma boa forma de visualizar as adaptações é realizando um 
confronto entre os processos e a ferramenta buscando introduzir no processo novas entradas que representam a nova ferramenta.

\section{UNIFICAÇÃO DO STAGE-GATE E FLUXO DE DESENVOLVIMENTO DE PRODUTO}

Neste contexto, as etapas do Stage-Gate (Figura 3) e o fluxo de desenvolvimento finalizado (Figura 4) foi unificado em uma nova DNP, conforme ilustra a Figura 5.

Figura 5 - Fluxograma DNP com aplicação da ferramenta Stage-Gate

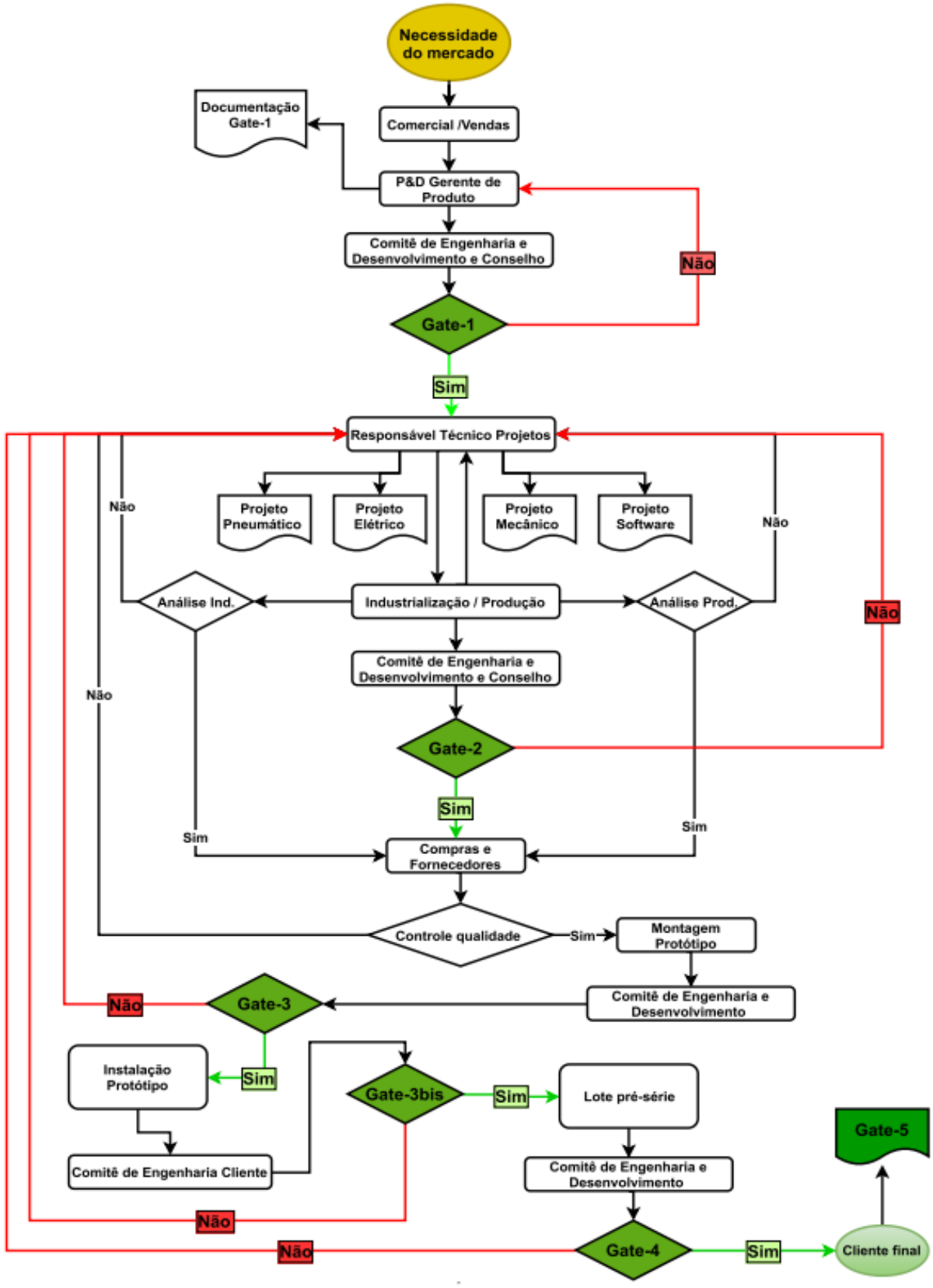

Fonte: Autoria própria (2019)

Foram agrupadas as atividades de utilizando ambos os documentos (Figura 5), sendo assim, foi possível identificar quais etapas do desenvolvimento do produto que devem ser finalizadas para permitir a conclusão e aprovação do 
Gate da sequência, permitindo assim que o projeto possa avançar para próxima fase do desenvolvimento que concluída permitirá o avanço para um novo Gate.

Para uma melhor compreensão das novas etapas agrupadas, foram detalhadas cada uma destas etapas em divisões individuais para validação dos resultados.

\section{VALIDAÇÕES DAS ESPECIFICAÇÕES DE PRODUTO GATE-1}

$\mathrm{Na}$ Figura 6 é listado de forma ilustrativa algumas entregas que proporcionaram a aprovação do Gate-1, entre elas estão à especificação técnica da máquina, analise de concorrência posição dentro da gama de produtos ou no mercado para máquina proposta, Análise Swot, ponto de partida para o projeto, previsão de custo/vendas e planificação das próximas atividades.

Figura 6 - Entregas do Stage-2 para etapa Gate-2

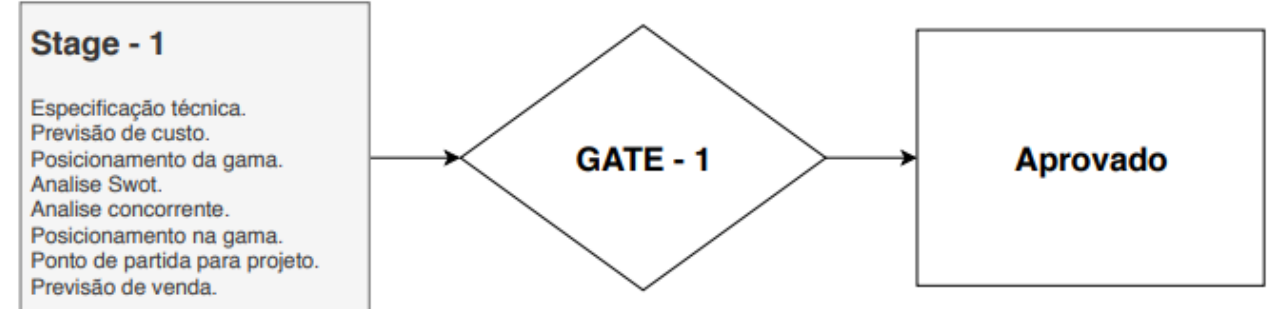

Fonte: Autoria própria (2019)

O Gate-1 apresentado na Figura 3 é uma importante etapa indispensável para o desenvolvimento de um novo produto, como base nas informações nele citadas o novo produto irá ganhar forma seja no desenvolvimento ou produção, em geral as informações fornecidas nesta etapa são confrontadas em todas as etapas da sequência.

\section{VALIDAÇÕES DO PROJETO E DEFINIÇÃO DO PROTÓTIPO GATE-2}

A validação do projeto se deu com a conclusão do projeto 3D e de todas as etapas necessárias para a confecção dos documentos que devem ser compilados dentro do Stage-1, permitindo que o seja feito a aprovação do Gate-2, na Figura 7 está representada as entregas necessárias para a aprovação do Gate-2.

Figura 7 - Entregas do Stage-2 para etapa Gate-2

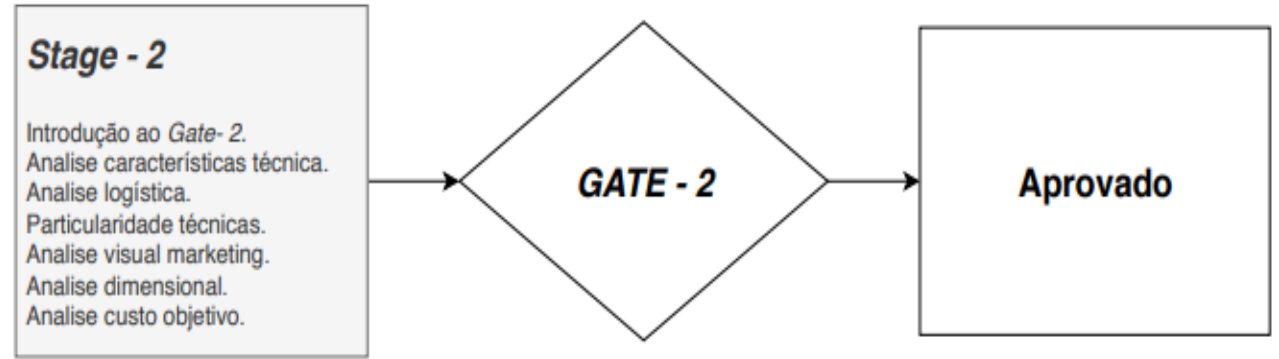

Fonte: Autoria própria (2019) 
Na Figura 7 podemos identificar as principais entregas do Stage-2 e que permitiu a aprovação do Gate-2 no presente projeto.

\section{VALIDAÇÕES DO PROTÓTIPO GATE-3}

A validação do protótipo se deu com a montagem da primeira máquina sendo apresentados todos os documentos pertinentes a esta etapa destacada na Figura 8, juntamente com a máquina montada que podemos verificar na Figura 9.

Figura 8 - Entregas do Stage-3 para etapa Gate-3

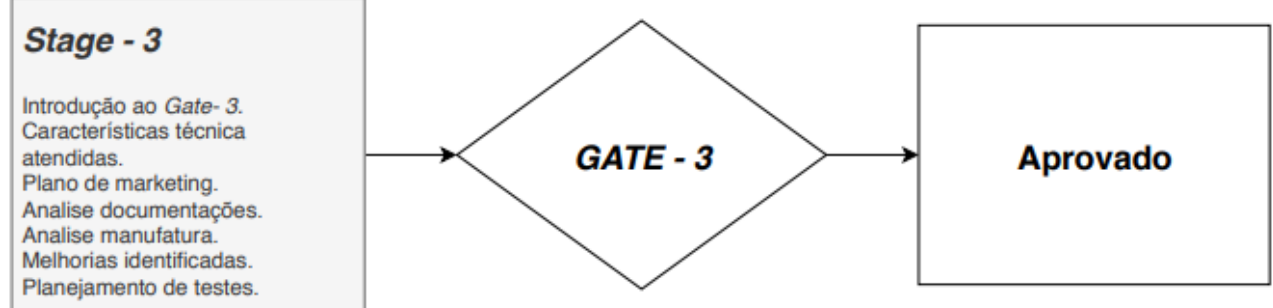

Fonte: Autoria própria (2019)

O Gate-3 representa é o momento que o produto já tem formato e pode ser observado e analisado de forma mais aprofundada, principalmente em relação a seus aspectos técnicos.

Figura 9 - Entregas do Stage-3 para etapa Gate-3

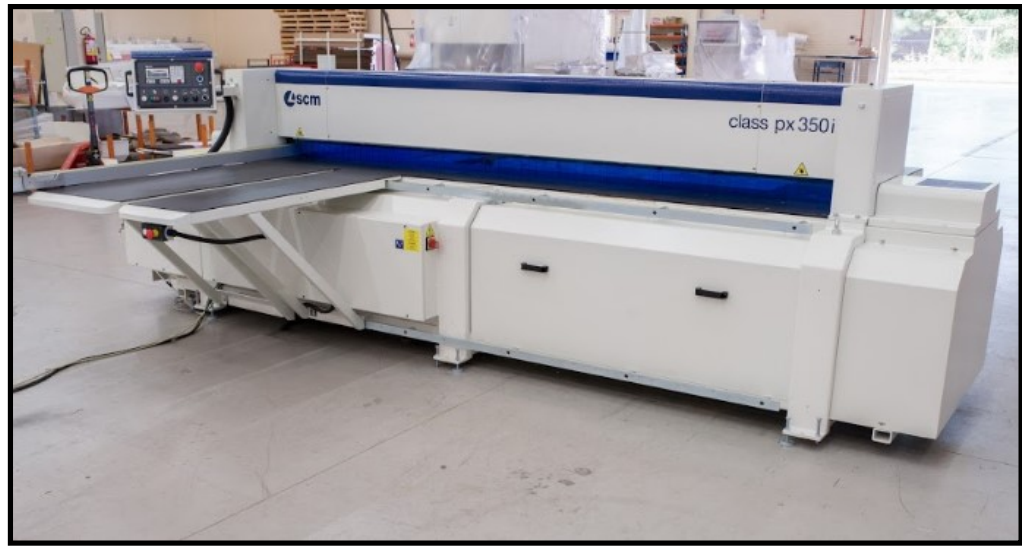

Fonte: SCM (2019)

A Figura 9 apresenta a máquina totalmente montada, sendo possível observar importantes aspectos como estética e qualidade na produção da máquina.

\section{VALIDAÇÕES DO PROTÓTIPO EM CAMPO GATE-3BIS}

A validação protótipo em campo se deu após três meses de utilização onde estavam presentes nessa validação os setores de Engenharia Mecânica, 
assistência técnica e o cliente. Na Figura 10 é representado os documentos de validação compilados para a realização do Gate-3bis é no cliente final.

Figura 10 - Entregas do Stage-3Bis para etapa Gate-3Bis

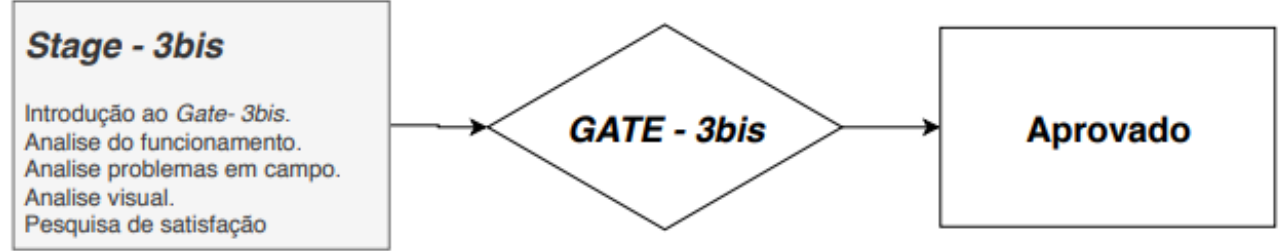

Fonte: Autoria própria (2019)

Esta validação do produto é realizada em campo e é uma importante etapa para se ter um parâmetro de referência em das expectativas do cliente final em relação a máquina.

\section{VALIDAÇÕES DO PROJETO GATE-4 E GATE-5}

As demais validações do projeto ainda não foram realizadas, pois atualmente a máquina está em processo de produção do lote pré-série ficando marcada a validação do projeto para fevereiro de 2020 . Na Figura 11 pode-se verificar a linha de montagem da máquina onde na ocasião está sendo montado o lote présérie.

Figura 11 - Máquina em produção pré-série

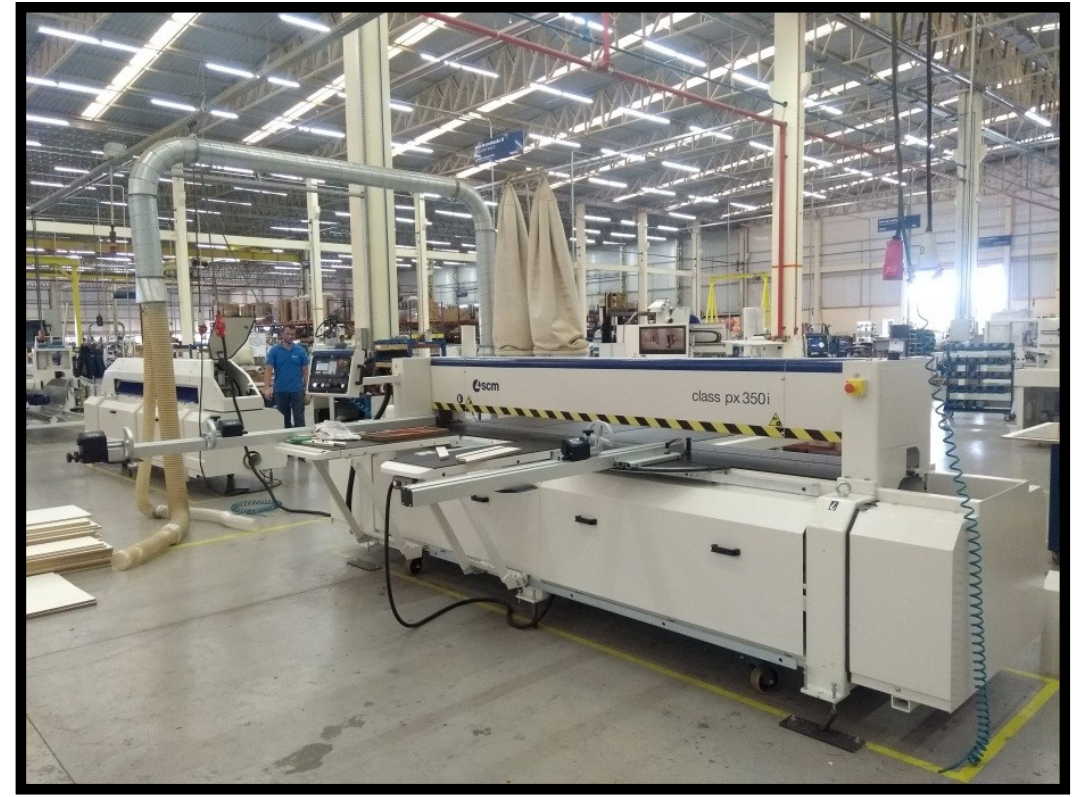

Fonte: Autoria própria (2019)

O momento de incluir uma determinada máquina em uma linha de produção é considerado uma das fases de ajuste de processos onde são identificadas melhorias para agilizar e dar mais confiabilidade na montagem da máquina, como 
podemos perceber na Figura 8, quando uma máquina é implementada em uma linha a mesma deve atender requisitos para que seja possível montar nesta mesma linha de montagem diferentes tipos de máquinas sem que seja interrompido o fluxo de montagem.

\section{CONCLUSÕES}

Com o presente estudo foi possível perceber a grande quantidade de processos envolvidos no gerenciamento de projetos de máquinas, sendo visível a relevância que uma ferramenta de gerenciamento de projetos como a StageGate pode proporcionar aos gestores.

Podemos então concluir que a utilização da ferramenta Stage-Gate teve grande contribuição dentro dos processos de desenvolvimento de produto aplicados no estudo de caso, tendo como aspecto principal para sua importância as etapas bem definidas e a utilização de documentos para aprovação dessas etapas, permitindo assim que se tenha um banco de dados de estrema importância para utilização em projetos de mesmas características técnicas e funcionais no futuro.

O estudo apresentou limitações no sentido de limitar-se a apenas ao estudo de uma indústria produtora de máquinas para fabricação de móveis, de médio porte. Encoraja-se futuras pesquisas no sentido de desenvolver estratégias de estudos semelhantes em outros contextos e ambientes, reportando os resultados ao ambiente estudado, possibilitando assim iniciativas de melhoria. 


\title{
Application of stage-gate in the management of machinery projects for furniture manufacturing industries
}

\begin{abstract}
Machinery manufacturers are small, medium or large, need to be prepared to give immediate answers with technological projects and short deadlines for the needs identified in the market. There are project management tools that can bring numerous benefits to companies in these areas. And the response time can determine the permanence of a company in the market or not. This work demonstrates the application of a project management methodology based on the Stage-Gate tool with documents related to PMBOK areas in the development of a specific machine for the furniture market, identified as a necessity for the world market. The work was based on a case study, in which it demonstrated the importance of the implementation of a methodology of project management for the development of machines for the furniture sector. The analysis of the application of this type of methodology allowed having a more comprehensive view of the project, being able to identify possible difficulties in advance, allowing the action and correction of eventual problems that would harm the result of the project.
\end{abstract}

KEYWORDS: Project Management. Stage-Gate. PMBOK. Furniture Manufacturing Industries. 


\section{REFERÊNCIAS}

ALVARES, F. T.; TAMBORELLI, H. W. V.; PEREIRA, M.; SANTOS, P. C.; RODRIGUES, S. J.; GODOI, H. C. Kaisen: o sucesso na estratégia de produção. Revista Cientifica, $11,2018$.

ALVES, J. N.; SILVA, T. B.; TAVARES, C. E. M.; DALSOLO, F. A utilização do Planejamento Estratégico como ferramenta de Gestão na Pequena Empresa. Revista da Micro e Pequena Empresa, 7, 2, 80-100, 2013.

BESANKO, O. D.; DRANOVE, D.; SHANLEY, M. Economics of Strategy. New York: Wiley, 2000.

BURGWAL, L. H. M. V.; RIBEIRO, C. D. S.; WALL, M. B. V.; CLAASSEN, E. Towards improved process efficiency in vaccine innovation: The vaccine innovation cycle as a validated, conceptual stage-gate model, 2018.

CASAROTTO, F. N.; SEVERINO, F. J.; ERNESTO, E. C. J. Gerênciamento de Projetos/ Engenharia Simultânea. 1 Edição, São Paulo, Editora Atlas, 1999.

COBAITO, C, F. Gerenciamento de Projetos: Na Ótica das Melhores Práticas do Stage-Gate e PMI. Ibero-american Jormal of Industrial Engineering, 5, 9,203-220, 2013. crossref

COOPER, R. G. Perspective: The Stage-Gate Idea-to-Launch Process-Update, What's New, and NexGen Systems. Product Innovation Management, 25, 213232, 2008 .

\section{crossref}

Costa, C, S, A., Santana, C, L., TRIGO, C, A. Qualidade do Atendimento ao Cliente: Um Grande Diferencial Competitivo para as Organizações. Revisata de Iniciação Cientifica (2015).

COUTINHO, L.; FERRAZ, J. C. Estudo da competitividade da indústria brasileira. Campinas: Papirus, 1994.

FROSI, F. O.; STEFFEN, C. Adaptando o Stage-Gate: Desenvolvimento de novos produtos com inovações incrementais na indústria de jogos digitais. Ministério da Ciência e Tecnologia. São Paulo, 2016.

GOMES, D. R.; SOUZA, S. D. C. Mapeamento do processo de produção em uma fábrica do polo de cerâmica vermelha do norte Fluminense. XXX Encontro nacional de engenharia de produção. São Carlos, SP, 2010. 
MELLO, E. B.; GANZER, P. P.; RASIA, I. C. R. B. R.; OLEA, P. M.; ROCHA, J. M. Processo de desenvolvimento de produtos e o sistema Stage-Gate. Gestão Contemporânea, Porto Alegre, Edição especial, 2012.

MELO, C, P, L. B.; OLIVEIRA, S. A.; SILVA, L. A.; PMBOK, O, R. Uma fonte de diretrizes no gerenciamento de projetos. Gestão Contemporânea, Tecnologia em Projeção, 8, 1, 2017.

PALUCH, S.; ANTONS, D.; BRETTEL, M.; HOPP, C.; SALGE, T.; O. PILLER, F.; WENTZEL, D. Stage-gate and agile development in the digital age: Promisses, perils, and boundary conditions. Journal of Business Research. 2019. crossref

PMI- Project Management Institute. [Acesso em 25 jun. 2019]. Disponível em: <https://brasil.pmi.org/brazil/home.aspx $>$.

RABECHINI, J. R.; CARVALHO, M. M.; LAURINDO, B. J. F. Fatores críticos para implementação de gerenciamento por projetos: o caso de uma organização de pesquisa. Revista produção. 12, 2, 2002.

RODRIGUES, R.; PAIXÃO, H. C. A Importância do Planejamento Estratégico na Gestão de Projetos. Congresso Nacional de Excelência em Gestão. 2016.

ROZENFELD, H.; AMARAL, D. C.; FORCELLINI, F. A.; TOLEDO, J. C.; SILVA, S. L.; ALLIPRANDINI, D. H.; SCALICE, R, K. Desenvolvimento de produtos: uma referência para a melhoria do processo. São Paulo: Saraiva, 2006.

SALGADO, E. G.; SALOMON, V. A. P.; MELLO, C. H. P.; FASS, F. D. M. Modelo de referência para desenvolvimento de produtos: Classificação, análise e sugestões para pesquisas futuras. Revista Produção Online, 2010.

crossref

SANTOS, P. J.; FORETI, D. J.; SANTOS, P. C.; CHAVES, C. E. L.; JUGER, P. A. Estudo de Caso: A Aplicação do Gerenciamento de Projetos para Entrar em uma Incubadora Universitária. Simpósio de excelência em Gestão e tecnologia. 2012.

SEVERO, E. A.; GUIMARÃES, J. C. F.; ROTTA, C.; TACCA, A.; DORION, E. C. H. Inovação de produto através do método Stage-Gate: Estudo de caso em uma indústria eletroeletrônica. Gestão Contemporânea, Porto Alegre, Edição especial, 2014.

SPEROTTO, F. Q. Setor moveleiro Brasileiro e gaúcho: características, configuração e perspectiva. Indicadores Econômicos FEE, Porto Alegre, 2018. 
Recebido: 12 Jul. 2019

Aprovado: 12 Out. 2020

DOI: 10.3895/gi.v16n4.10358

Como citar:

CIESLINSKI, V. et al. Aplicação da ferramenta stage-gate no gerenciamento de projetos de máquinas para Indústrias moveleiras. R. Gest. Industr., Ponta Grossa, v. 16, n. 4, p. 57-75, Out./Dez. 2020. Disponível em: https://periodicos.utfpr.edu.br/revistagi

Correspondência:

Vanderlei Cieslinski

Universidade da Região de Joinville (UNIVILLE), São Bento do Sul, Santa Catarina, Brasil.

Direito autoral: Este artigo está licenciado sob os termos da Licença Creative Commons-Atribuição 4.0

Internacional.

(c) (1) 